ORIGINAL ARTICLE

\title{
Patterns of diffusion of evidence-based clinical programmes: a case study of the Hospital Elder Life Program
}

\author{
Elizabeth H Bradley, Tashonna R Webster, Mark Schlesinger, Dorothy Baker, Sharon K Inouye
}

Qual Saf Health Care 2006;15:334-338. doi: 10.1136/qshc.2006.018820

See end of article for authors' affiliations .....................

Correspondence to: E H Bradley, Department of Epidemiology and Public Health, Yale School of Medicine, 60 College Street, New Haven, CT 06520-8034, USA elizabeth.bradley@yale. edu

Accepted 30 July 2006

\begin{abstract}
Background: The effective translation of scientific evidence into clinical practice is paramount to improving the quality and safety of patient care. However, little is known about the patterns of diffusion of evidencebased programmes in healthcare.

Objectives: To study the pattern of diffusion of an evidence-based programme to improve the quality and safety of care for hospitalised older adults.

Methods: The diffusion of the Hospital Elder Life Program (HELP), a multifaceted programme to reduce delirium in hospitalised adults, was examined. Using a survey of all hospitals that contacted the HELP Dissemination Project for more than 2 years, the proportion of hospitals that adopted the programme, the programme fidelity to the original design in terms of structure and process, and the perceived reasons for non-adoption were identified.

Results: Programme fidelity was highest among structural features (eg, staffing levels); programme modifications were more commonplace in processes of care (eg, the participation of volunteers in patient care interventions). Senior management support and the programme expense were the most commonly cited reasons for non-adoption of HELP.

Conclusion: Diffusion and take-up rates for this evidence-based programme were substantial; however, programme fidelity was not complete and some hospitals did not adopt the programme at all. Clinicians, researchers and funding agents seeking to promote effective translation of research should be realistic about diffusion rates and recognise the critical ingredient of senior management support to propel adoption of evidence-based programmes to improve quality and safety.
\end{abstract}

E ffective translation of scientific evidence into clinical practice is of paramount importance to ensure that patients benefit from scientific research. Recent policies and funding priorities ${ }^{12}$ for the National Institutes of Health and the Agency for Healthcare Research and Quality reflect this focus on translating research into practice. Despite substantial efforts to speed the diffusion of evidence-based practices, ${ }^{34}$ previous studies have not tracked the national dissemination of a clinical programme to identify common patterns of diffusion. Information on patterns of diffusion can help researchers and funding agencies anticipate not only the pace of adoption but also the adaptations that occur during the diffusion process, as greater effort is devoted to translating science into practice.

Accordingly, we sought to study the pattern of diffusion of an evidence-based programme to improve care for hospitalised older adults as a case study of the phenomenon of translating research evidence into practice. The Hospital Elder Life Program (HELP) $)^{5}$ has been shown to be both effective $^{56}$ and cost-effective ${ }^{78}$ in reducing episodes of delirium and functional decline in hospitalised older adults. On the basis of this evidence, substantial efforts ${ }^{9-11}$ have been made to replicate the HELP in hospitals nationally. However, previous studies have not examined the pattern of its national diffusion and success in national dissemination.

We used as a case study the HELP Dissemination Project, a national programme to support hospitals adopting HELP. This project, funded by federal and foundation sources, provides training materials, an interactive website ${ }^{12}$ and support from experienced staff in establishing a new HELP site. For this study, we surveyed staff members in hospitals who had contacted the HELP Dissemination Project team or registered for the HELP special interest group meetings at the American Geriatrics Society and Gerontological Society of America Annual Meetings about the experience of programme adoption at their hospitals. Understanding the process of diffusion of HELP can be useful as a case study to identify common patterns in the diffusion of evidencebased clinical programmes.

\section{METHODS}

\section{Study design and sample}

We conducted a study of all hospitals that had expressed interest in implementing HELP by contacting the HELP dissemination team ${ }^{12}$ or by participating in HELP special interest groups at the American Geriatrics Society or Gerontological Society of America Annual Meetings in the 2 years before the survey, which was conducted in JanuaryMay 2005. The research procedures were approved by the institutional review board at the Yale School of Medicine, New Haven, Connecticut, USA.

\section{Data collection}

We used an online survey directed to the hospital staff members who had made contact with the HELP dissemination team or had participated in the HELP special interest groups. Respondents were surveyed during January-May 2005, which was at least 6 months after their original contact with the dissemination project. The questionnaire asked respondents to characterise their programming for delirium

Abbreviation: HELP, Hospital Elder Life Program 
in one of the following categories of diffusion and programme adoption:

- the hospital was implementing HELP as a contracted HELP dissemination site-that is, with access to all copyrighted training materials and protocols, assistance from the HELP dissemination team, and protected parts of the HELP website;

- the hospital was implementing a programme that was considered to be similar to HELP but without access to copyrighted protocol, training materials and protected parts of the HELP website;

- the hospital had clinical guidelines or pathways for delirium but no additional programming for delirium; or

- the hospital was implementing none of the above.

All respondents were asked to indicate from a predetermined set of reasons, which were developed based on our previous qualitative research, ${ }^{9}{ }^{10}$ why HELP had or had not been adopted at their hospital.

For all hospitals that characterised themselves as either a contracted HELP dissemination site or a hospital with a programme similar to HELP, we ascertained programme fidelity to the original HELP design. ${ }^{5}$ Programme fidelity was evaluated with respect to (1) staffing levels and (2) processes of care. Staffing levels were measured by (a) the type of staff associated with the programme and (b) the hours per week of each staff type associated with the programme. Process of care was measured by (a) reported implementation of the six core interventions of HELP, (b) the use of volunteers in these interventions and (c) the use of interdisciplinary rounds. The six core interventions included daily orientation and therapeutic activities, aiding sleep (ie, a non-pharmacological sleep protocol and sleep enhancement programme), early mobilisation (ie, ambulation or range of motion exercises), visual aids and reminders to use glasses, hearing aids and reminders to use aids, and dehydration protocol (ie, recognition and volume repletion).5 Lastly, we evaluated the perceived effect of the programme on 22 patient and staff outcomes, which were developed on the basis of our previous qualitative work ${ }^{9}$ as potential ways in which the programme might influence patient care and staff experiences.

\section{Data analysis}

We used standard descriptive statistics to describe the proportion of hospitals with the following various types of adoption: (1) a contracted HELP dissemination site, (2) a similar programme but not a contracted HELP dissemination site, (3) clinical guidelines or pathways for delirium only or (4) none of these. We examined the level of programme fidelity of the contracted HELP sites and similar programmes relative to the original HELP protocol published by Inouye et al. ${ }^{5}$ We used t tests and $\chi^{2}$ statistics as appropriate to evaluate whether there were significant differences in programme fidelity and perceived programme effect across hospitals of different adoption types - that is, as contracted HELP sites compared with sites that adopted a similar programme but not as a contracted HELP site. The criterion for significance was $\alpha<0.05$. All analyses were carried out using SAS V.9.1.

\section{RESULTS}

\section{Sample characteristics}

A total of 105 people were eligible for our survey on HELP implementation. Of these, $24(19.2 \%)$ did not have valid contact information and could not be located. Of the remaining 81 people whom we could locate, $63(77.8 \%)$ responded to the survey. About $79 \%$ of the responding hospitals were teaching hospitals, $76 \%$ were part of a multihospital system and $89 \%$ were non-profit organisations (table 1).

\section{Contracted HELP dissemination sites: first-hand dissemination}

Among the 63 respondent hospitals, 16 (25.4\%) had implemented the programme as a contracted HELP dissemination site, which included having access to copyrighted protocols and training materials, as well as web-based support (fig 1). We term these hospitals "first-hand" dissemination sites because they had the closest contact and engagement with the HELP dissemination team. In these hospitals, programme fidelity (table 2) compared with the original programme designed by Inouye et $a l^{56}$ was high, although not complete. All 16 of the first-hand dissemination sites had an elder life nurse specialist, who devoted an average of about $26 \mathrm{~h} /$ week to HELP. Three quarters of these hospitals had an elder life specialist or programme coordinator, who on average devoted $36 \mathrm{~h} /$ week to HELP, and nearly all these hospitals had a geriatrician working with the programme, with an average of $2.5 \mathrm{~h} /$ week devoted to the programme. Most first-hand dissemination sites also reported carrying out all or almost all of six core interventions of HELP, and all sites had volunteers participating in patient care interventions, as recommended in the programme protocols. Nonetheless, even in first-hand dissemination sites, local adaptations occurred. For instance, not all hospitals implemented all six interventions, and some did not use the interdisciplinary team rounds as prescribed in the original programme. We found no significant differences between the contracted HELP sites and the sites with similar programmes in the presence or types of staff involved, including hours per week of geriatrician time; however, contracted HELP sites were significantly $(\mathrm{p}<0.05)$ less likely than sites with similar programmes to have interdisciplinary rounds (table 2 ).

\section{Sites adopting programmes similar to HELP: second- hand dissemination}

An additional $13(20.6 \%)$ respondents reported that their hospitals had not become a contracted HELP dissemination site but had nonetheless started programmes that they characterised as similar to HELP (fig 1). Of these hospitals that had implemented a programme similar to HELP, reported programme fidelity (table 2) was high for staffing levels and most processes of care. In fact, in the six core interventions, reported implementation was higher for "second-hand" than for first-hand dissemination hospitals, although the difference was not significant. However, we observed significant differences in some process-of-care

\begin{tabular}{|ll|}
\hline $\begin{array}{l}\text { Table } 1 \text { Characteristics of participating } \\
\text { hospitals }(\mathrm{n}=63)\end{array}$ \\
\hline & $\mathbf{n}(\%)$ \\
\hline $\begin{array}{l}\text { Teaching hospital } \\
\text { Yes }\end{array}$ & $50(79)$ \\
No & $13(21)$ \\
Hospital type & $56(89)$ \\
Non-profit & $4(6)$ \\
For profit & $3(5)$ \\
Missing & $48(76)$ \\
Multihospital system & $15(24)$ \\
Yes & $11(17)$ \\
No & $32(51)$ \\
No of staffed beds & $20(32)$ \\
$<200$ & \\
$200-599$ & \\
$>600$ & \\
\hline
\end{tabular}




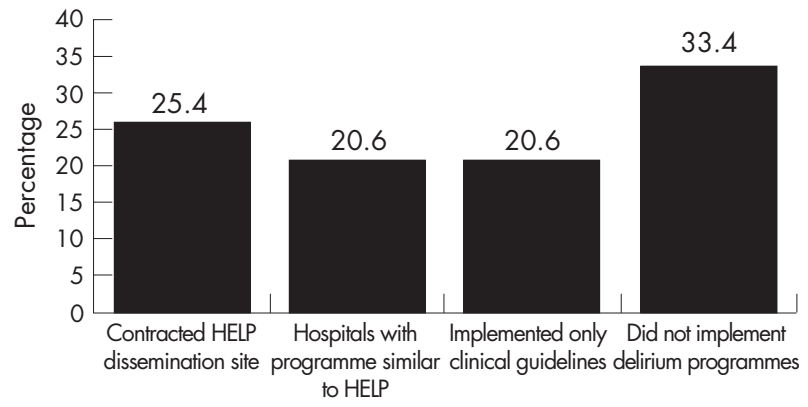

Figure 1 Proportion of hospitals by stage of diffusion and adoption. HELP, Hospital Elder Life Program.

measures. Compared with contracted HELP dissemination sites (ie, first-hand dissemination hospitals), second-hand dissemination hospitals were significantly more likely to conduct interdisciplinary rounds, and they included volunteers in significantly fewer patient-care interventions.

\section{Other hospitals}

Another 13 (20.6\%) hospitals reported that they had developed clinical guidelines or pathways for delirium but had not implemented HELP or a similar programme (fig 1). Respondents from the remaining 21 (33.4\%) hospitals reported that their hospital had no clinical programming that targeted delirium. We observed no significant differences in hospital size, the proportion that was part of a multihospital system, ownership type (ie, for profit or nonprofit), or teaching status across the four levels of diffusion. The most commonly reported reasons for non-adoption of HELP were a lack of senior management support, the programme expense and a lack of doctor and nurse leaders for such a programme (table 3 ).

\section{Perceived effect of the programme}

Despite some differences in levels of programme fidelity between the first-hand and second-hand dissemination sites, the perceived effect on patients and staff outcomes did not differ significantly by type of dissemination site (table 4 ). In most cases the perceived effect of the programme was substantial, with respondents for typically two thirds or more of the hospitals reporting that the programme had either a little or a lot of impact on many positive patient and staff outcomes.

\section{DISCUSSION}

We found that nearly half the hospitals that expressed initial interest in HELP during a 2-year period of active dissemination ultimately adopted HELP or a similar programme as of May 2005, when our study ended. Additionally, several hospitals had developed clinical guidelines or pathways for delirium even if they had not implemented HELP or a similar programme. Although this rate of adoption is substantial, considering the $90 \%$ failure rates of most innovations, ${ }^{13}$ the the findings suggest that realism is important in diffusing evidence-based innovations, as many potential adopting sites may not ultimately take up the new programme despite their initial inquiries and interest. Unlike many clinical innovations that focus on new technology, drugs or medical procedures, HELP is a complex innovation that is labourintensive and requires coordination across multiple disciplines. As has been suggested before, ${ }^{914}$ such human technologies can present challenges for adopting sites seeking to maintain programme fidelity.

Our findings also disclose information about patterns of adaptation of evidence-based programmes as they diffuse.
Table 2 Programme fidelity among contracted Hospital Elder Life Program dissemination sites and hospitals adopting a similar programme

\begin{tabular}{|c|c|c|}
\hline & $\begin{array}{l}\text { Contracted HELP } \\
\text { dissemination sites }\end{array}$ & $\begin{array}{l}\text { Hospitals } \\
\text { adopting a similar } \\
\text { programme }\end{array}$ \\
\hline \multicolumn{3}{|l|}{ Staffing levels } \\
\hline $\begin{array}{l}\text { Elder life nurse } \\
\text { specialist (\%) }\end{array}$ & 100 & 92.3 \\
\hline Mean (SD) h/week & 25.7 (14.4) & $27.0(22.5)$ \\
\hline $\begin{array}{l}\text { Elder life specialist } \\
\text { /programme coordinator (\%) }\end{array}$ & 75 & 53.9 \\
\hline Mean (SD) h/week & $36.0(39.5)$ & $18.7(19.1)$ \\
\hline Geriatrician (\%) & 87.5 & 69.2 \\
\hline Mean (SD) h/week & $2.5(3.1)$ & $11.2(20.3)$ \\
\hline \multicolumn{3}{|l|}{$\begin{array}{l}\text { Sites with interventions } \\
\text { implemented }\end{array}$} \\
\hline $\begin{array}{l}\text { Daily orientation and } \\
\text { therapeutic activities (\%) }\end{array}$ & 100 & 100 \\
\hline Aiding sleep (\%) & 75.0 & 84.6 \\
\hline Early mobilisation (\%) & 93.8 & 100 \\
\hline $\begin{array}{l}\text { Visual aids and reminders } \\
\text { to use glasses (\%) }\end{array}$ & 93.8 & 100 \\
\hline $\begin{array}{l}\text { Hearing aids and reminders } \\
\text { to use aids (\%) }\end{array}$ & 93.8 & 100 \\
\hline Dehydration protocol (\%) & 87.5 & 100 \\
\hline Interdisciplinary rounds (\%)* & 56.3 & 92.3 \\
\hline $\begin{array}{l}\text { Mean (SD) no of interventions } \\
\text { in which volunteers are } \\
\text { involved** }\end{array}$ & s $4.8(2.0)$ & $1.9(2.2)$ \\
\hline
\end{tabular}

HELP, Hospital Elder Life Program. ${ }^{*} \mathrm{p}<0.05$.

Table 3 Reported reasons for non-adoption of Hospital Elder Life Program or a similar programme $(n=32$ hospitals that were non-adopters)

\begin{tabular}{ll}
\hline & $\mathbf{n}^{\star}(\%)$ \\
\hline Lack of support from senior management & $17(53 \%)$ \\
Too expensive & $13(41 \%)$ \\
No nurse leader & $9(28 \%)$ \\
No doctor leader & $8(25 \%)$ \\
Lack of support from nurses & $7(22 \%)$ \\
Chose another programme instead & $6(19 \%)$ \\
Lack of support from doctors & $6(19 \%)$ \\
Not consistent with the organisation's mission & $4(13 \%)$ \\
\hline
\end{tabular}

*Percentages add to more than $100 \%$ because respondents could report more than one reason.

The results indicated that programme fidelity was generally high among first-hand dissemination sites with strong links to the original HELP dissemination team, although some aspects (ie, aiding sleep intervention and use of interdisciplinary rounds) were less fully implemented. This programme fidelity was apparent across a range of hospitals, diverse in terms of size and teaching status. Our previous research $^{10}$ has suggested that internal aspects of the organisation, such as the administrative and clinical support for the innovation, rather than attributes related to size and teaching status, are central in anticipating the degree of programme adoption and replication.

In addition to the high programme fidelity among the contracted HELP dissemination sites, there was also generally high programme fidelity among the sites that adopted a similar programme but without access to all the protocols and training materials of HELP. However, these second-hand dissemination sites did modify certain processes of care to fit local circumstances. Such adaptations were most apparent not in staffing levels or in implementation of key interventions, but rather in the ways in which interventions were 
Table 4 Reported effect of Hospital Elder Life Program or similar programmes

\begin{tabular}{|c|c|c|c|c|c|c|}
\hline \multirow[b]{2}{*}{ Area of impact } & \multicolumn{3}{|c|}{ Contracted HELP dissemination sites* } & \multicolumn{3}{|c|}{ Hospitals adopting a similar programme* } \\
\hline & $\begin{array}{l}\text { A lot of impact, } \\
\text { n (\%) }\end{array}$ & $\begin{array}{l}\text { A little impact, } \\
\text { n (\%) }\end{array}$ & $\begin{array}{l}\text { No impact, } \\
\text { n (\%) }\end{array}$ & $\begin{array}{l}\text { A lot of impact, } \\
\text { n (\%) }\end{array}$ & $\begin{array}{l}\text { A little impact, } \\
\text { n (\%) }\end{array}$ & $\begin{array}{l}\text { No impact, } \\
\text { n (\%) }\end{array}$ \\
\hline Reduced length of stay & $4(40.0)$ & $4(40.0)$ & $2(20.0)$ & $5(50.0 \%)$ & $4(40.0)$ & $1(10.0)$ \\
\hline Reduced cost/patient & $4(50.0)$ & $3(37.5)$ & $1(12.5)$ & $2(25.0 \%)$ & $5(62.5)$ & $1(12.5)$ \\
\hline Decreased episodes of delirium & $8(80.0)$ & $2(20.0)$ & - & $5(50.0 \%)$ & $5(50.0)$ & - \\
\hline Reduced falls & $4(40.0)$ & $5(50.0)$ & $1(10.0)$ & $5(45.5)$ & $6(54.5)$ & - \\
\hline Reduced use of restraints & $4(40.0)$ & $6(60.0)$ & - & $4(40.0)$ & $5(50.0)$ & $1(10.0)$ \\
\hline Reduced use of sitters or companions & $4(44.4)$ & $5(56.6)$ & - & $3(37.5)$ & $4(50.0)$ & $1(12.5)$ \\
\hline Reduced discharges to nursing homes & $2(20.0)$ & $7(70.0)$ & $1(10.0)$ & $2(18.2)$ & $5(45.5)$ & $4(36.4)$ \\
\hline Reduced functional decline & $6(60.0)$ & $3(30.0)$ & $1(10.0)$ & $7(63.6)$ & $4(36.4)$ & - \\
\hline Increased patient satisfaction & 11 (91.7) & $1(8.3)$ & - & $9(90.0)$ & $1(10.0)$ & - \\
\hline Increased nurses' job satisfaction & $8(66.7)$ & $4(33.3)$ & - & $6(60.0)$ & $3(30.0)$ & $1(10.0)$ \\
\hline Increased nurse retention & $2(40.0)$ & $1(20.0)$ & $2(40.0)$ & $2(25.05)$ & $4(50.0)$ & $2(25.0)$ \\
\hline Increased doctors' job satisfaction & $1(20.0)$ & $3(60.0)$ & $1(20.0)$ & $3(42.9)$ & $3(42.9)$ & $1(14.2)$ \\
\hline Reduced incontinence & - & $5(71.4)$ & $2(28.6)$ & $2(22.2)$ & $4(44.5)$ & $3(33.3)$ \\
\hline Reduced pressure ulcers & - & $4(57.1)$ & $3(42.9)$ & $4(44.5)$ & $3(33.3)$ & $2(22.2)$ \\
\hline Reduced unscheduled readmissions & - & $3(75.0)$ & $1(25.0)$ & $2(25.0)$ & $5(62.5)$ & $1(12.5)$ \\
\hline Creation of delirium guidelines & 3 (33.3) & $3(33.3)$ & $3(33.3)$ & $6(60.0)$ & - & $4(40.0)$ \\
\hline Nurses' knowledge of geriatrics & $7(53.9)$ & $5(38.4)$ & $1(7.7 \%)$ & $7(63.6)$ & $4(36.4)$ & - \\
\hline Nurse aids' knowledge of geriatrics & $4(30.8)$ & $8(61.5)$ & $1(7.7)$ & $4(40.0)$ & $5(50.0)$ & $1(10.0)$ \\
\hline Doctors' knowledge of geriatrics & $1(8.3)$ & $9(75.0)$ & $2(16.7)$ & $3(33.3 \%)$ & $6(66.7)$ & - \\
\hline Residents' knowledge of geriatrics** & $1(8.3)$ & $9(75.0)$ & $2(16.7)$ & $6(60.0)$ & $4(40.0)$ & - \\
\hline Pharmacists' knowledge of geriatrics & 3 (33.3) & $3(33.3)$ & 3 (33.3) & $5(50.0)$ & $3(30.0)$ & $2(20.0)$ \\
\hline Therapists' knowledge of geriatrics & $3(27.3)$ & $7(63.6)$ & $1(9.1)$ & $5(45.5)$ & $6(54.6)$ & - \\
\hline
\end{tabular}

implemented, such as the degree to which volunteers participated in patient-care interventions. The extensive inclusion of volunteers in patient-care interventions may require a change in mindset among clinicians and administrators at the hospital, as well as increased coordination with hospital departments, including the volunteer or social services departments. The fact that we found less programme fidelity in this aspect of the programme is consistent with broader literature on diffusion, ${ }^{15}$ which suggests that adaptations are typically more common in areas that require substantial culture shift from the status quo and require new coordination efforts.

Interestingly, there were no significant differences between the reported effect of the programmes in the first-hand and second-hand dissemination sites. This may be due to the selfreporting of outcomes and the desire to report a positive effect of the programme. In addition, however, it may signal that the adaptations were in fact important to achieving desired programme effectiveness in the local settings of the hospitals, at least as perceived by the staff most involved in the programme. We could not assess the degree to which the adaptations might compromise or improve the ability to accomplish the objective outcomes of the programme; however, the data suggest that such adaptations did not compromise the effect of the programme as perceived by the adopting hospital. Experts in diffusion in healthcare ${ }^{16}$ have suggested, in fact, that programme adaptations and modification may be essential to accomplishing the desired goals of the adopters, although these may differ in some respects from the goals of the original programme innovators.

The results of the study should be considered in the light of several issues. All data are self-reported and are therefore subject to the recall and reporting bias that is inherent in such reports. For example, the first-hand dissemination HELP sites typically monitor and track intervention closely, and thus their reports may be data-based and more objective, whereas other sites may have based their reports on more subjective information. Therefore, reported staffing, processes and effects may differ if validated with more comprehensive and objective data. In addition, we had a relatively limited sample size. Nevertheless, we did survey all hospitals that were officially implementing HELP during the study year; hence, the sample is relatively comprehensive for the objectives of the inquiry. Lastly, we surveyed only one person per hospital, and other staff in the hospital may have had differing views regarding how and why a programme was implemented. However, we did interview the person who was most involved in HELP or similar programme implementation and thus was hopefully most knowledgeable about the day-to-day operation of the programme.

Despite the national calls to improve the translation of research into practice, diffusing even evidence-based clinical innovations is a daunting task. In disclosing these challenges in the context of disseminating HELP, several lessons learnt about translating research into practice and programme diffusion emerged. These lessons have been particularly helpful in continuing the administration and spread of HELP nationally and also suggest more general insights about translating evidence-based but complex programmes into practice. Firstly, about one quarter of the sites that expressed initial interest ultimately implemented HELP. This proportion would have been higher if we had excluded from the study participants who were personally interested in HELP but were not in a position or empowered to promote programme adoption at their institutions. Nevertheless, the finding suggests that researchers and funding agencies need to acknowledge the limits on quickly translating research into widespread practice. Secondly, an almost equal number of sites expressing initial interest in HELP did not implement HELP but did implement what they considered a similar programme targeting delirium prevention and improving care for older adults. Therefore, dissemination efforts may be important catalysts for other programmatic improvement even if they do not result in the direct replication of the original programme. Thirdly, as the programme diffused, fidelity to the original specifications of the programme was imperfect. Adaptations occurred in core aspects of the programme (eg, use of volunteers, aiding sleep interventions, 
and interdisciplinary rounds). Although the effectiveness of a replicated HELP program with adaptations has been shown, ${ }^{11}$ the effectiveness of other modified programmes similar to HELP has not been formally evaluated. Therefore, it is difficult to anticipate the effect of innovations as they diffuse with programmatic modifications. Future research assessing the connection between programme fidelity and its effectiveness and sustainability over the longer term would benefit organisations adopting it. Fourthly, this and previous studies $^{10}$ 17-19 $^{19}$ have identified the importance of senior management support, clinical leadership and perceived value in implementing innovations, even those that have strong evidence bases. Our findings highlight the importance of organisational contextual elements, including leadership and perceived expense, in the adoption of innovative clinical programmes. Clinicians, researchers and funding agents seeking to promote effective translation of patient care improvements need to be aware of and plan how to navigate these organisational features to create and sustain positive changes in care.

\section{Authors' affiliations \\ E H Bradley, T R Webster, Department of Epidemiology and Public Health, Yale School of Medicine, New Haven, Connecticut, USA \\ S K Inouye, Aging Brain Center, Hebrew Seniorlife, Boston, \\ Massachusetts, USA \\ M Schlesinger, Department of Epidemiology and Public Health, Yale School of Medicine, New Haven, Connecticut, USA \\ D Baker, Department of Internal Medicine, Section of Geriatrics, Yale School of Medicine, USA}

Funding: This research was supported by grants from the Commonwealth Fund (numbers 20000484 and 990912), the Retirement Research Foundation (number 99-361), the Alzheimer's Foundation of America, The Institute for Incentives in Health Care, The National Library of Medicine (G08LM08085) and the Claude D Pepper Older Americans Independence Center at Yale (number P30AG21342). EHB is supported in part by the Patrick and Catherine Weldon Donaghue Medical Research Foundation (no DF02-102 and no DF98-105). SKI is also supported partly by grants from the National Institute on Aging (numbers R21AG025193 and K24AG00949).

Competing interests: None.

\section{REFERENCES}

1 Agency for Healthcare Research and Quality. Special emphasis notice: research priorities for the Agency for Healthcare Research and Quality. http://grants2.nih.gov/grants/guide/notice-files/NOT-HS-05-005.html (accessed 24 Feb 2006).

2 National Center for Research Resources. Institutional Clinical and Translational Science Awards. http://www.ncrr.nih.gov/clinicaldiscipline.asp (accessed 20 Mar 2006).

3 Farquhar CM, Stryer D, Slutsky J. Translating research into practice: the future ahead. Int J Qual Health Care 2002; 14:233-49.

4 Bradley EH, Webster TR, Baker D, et al. Translating research into practice: speeding the adoption of innovative health care programs. Issue Brief (Commonw Fund) 2004;724:1-12.

5 Inouye SK, Bogardus ST Jr, Baker DI, et al. The Hospital Elder Life Program: a model of care to prevent cognitive and functional decline in older hospitalized patients. J Am Geriatr Soc 2000;48:1697-706.

6 Inouye SK, Bogardus ST Jr, Charpentier PA, et al. A multicomponent intervention to prevent delirium in hospitalized older patients. N Engl J Med 1999;340:669-76.

7 Rizzo JA, Bogardus ST Jr, Leo-Summers L, et al. Multicomponent targeted intervention to prevent delirium in hospitalized older patients: what is the economic value? Med Care 2001;39:740-52.

8 Leslie DL, Zhang Y, Bogardus ST, et al. Consequences of preventing delirium in hospitalized older adults on nursing home costs. J Am Geriatr Soc 2005:53:405-9.

9 Bradley EH, Schlesinger M, Webster TR, et al. Translating research into clinical practice: making change happen. J Am Geriatr Soc 2004;52:1875-82.

10 Bradley EH, Webster TR, Baker D, et al. After adoption: sustaining the innovation. A case study of disseminating the hospital elder life program. J Am Geriatr Soc 2005;53:1455-61.

11 Rubin FH, Williams JT, Lescisin DA, et al. Replicating the Hospital Elder Life Program (HELP) in a community hospital and demonstrating effectiveness using administrative data. J Am Geriatr Soc 2006;54:969-74.

12 Yale University School of Medicine. The Hospital Elder Life Program. http:// elderlife.med.yale.edu/public/public-main.php (accessed 7 Mar 2006).

13 Skinner J, Staiger D. Technology adoption from hybrid corn to beta-blockers, NNER working paper 11251. Cambridge, MA: National Bureau of Economic Research, 2005.

14 Rogers EM. Diffusion of innovations. New York: Free Press, 1995.

15 Rogers EM. Lessons for guidelines from the diffusion of innovations. It Comm J Qual Improv 1995;21:324-8.

16 Greenhalgh T, Robert G, Macfarlane F, et al. Diffusion of innovations in service organizations: systematic review and recommendations. Milbank $Q$ 2004;82:581-629.

17 Weiner BJ, Shortell SM, Alexander J. Promoting clinical involvement in hospital quality improvement efforts: the effects of top management, board, and physician leadership. Health Serv Res 1997;32:491-510.

18 Shortell SM. Increasing value: a research agenda for addressing the managerial and organizational challenges facing health care delivery in the United States. Med Care Res Rev 2004;61:12S-30S.

19 Berwick DM. Curing health care: new strategies for quality improvement: a report on the National Demonstration Project on Quality Improvement in Health Care, 1 st edn. San Francisco, CA: Jossey-Bass, 1990.

\section{bmjupdates+}

bmjupdates+ is a unique and free alerting service, designed to keep you up to date with the medical literature that is truly important to your practice.

bmjupdates+ will alert you to important new research and will provide you with the best new evidence concerning important advances in health care, tailored to your medical interests and time demands.

Where does the information come from?

bmjupdates+ applies an expert critical appraisal filter to over 100 top medical journals A panel of over 2000 physicians find the few 'must read' studies for each area of clinical interest

Sign up to receive your tailored email alerts, searching access and more...

www.bmjupdates.com 